\title{
Fluid dynamic characterisation of a laboratory scale rocked bag
}

\section{bioreactor}

Douglas T. J. Marsh ${ }^{\mathrm{a}}$, Akinlolu O. O. Odeleye ${ }^{\mathrm{a}, \mathrm{b}}$, Andrea Ducci ${ }^{\mathrm{c}}$, Matthew D. Osborne ${ }^{\mathrm{d}}$, Gary J. Lye $^{\mathrm{a}}$ and Martina Micheletti ${ }^{\mathrm{a}}$

aThe Advanced Centre for Biochemical Engineering, Department of Biochemical Engineering, University College London, Gordon Street, London, WC1H 0AH, U.K.

${ }^{\mathrm{b}}$ Institute of Biomedical Engineering, University of Oxford, Old Road Campus Research Building, Headington, Oxford, OX3 7DQ, U.K.

${ }^{\mathrm{c}}$ Department of Mechanical Engineering, University College London, Torrington Place, London, WC1E 7JE, U.K.

${ }^{\mathrm{d} E l i}$ Lilly S.A. Irish Branch, Dunderrow, Kinsale, Co. Cork, Ireland.

\begin{abstract}
Single-use technology is being widely adopted for the manufacture of biotherapeutics and cell therapy products. Rocked single-use bioreactors in particular have been commonly used, however the hydrodynamics have rarely been characterised and are poorly understood. In this work, phase-resolved Particle Image Velocimetry and high frequency visual fluid tracking were used to investigate the flow pattern and velocity characteristics for the first time. The studies were performed on an optically accessible mimic of a Sartorius 2L CultiBag at different conditions. Wave formation was observed and higher rocking speeds caused the fluid to move proportionately out of phase with respect to the platform. Dimensional comparisons of fluid velocities with conventional bioreactors suggest that similar fluid dynamics characteristics can be achieved between rocked and stirred configurations. These results provide a first insight into the fluid dynamics of a novel bioreactor type at relevant process conditions supporting the generation of scale translation laws.
\end{abstract}


Topical Area: Transport Phenomena and Fluid Mechanics

Keywords: mixing, single-use, bioreactor, wave, Particle Image Velocimetry (PIV)

\section{Introduction}

Monoclonal antibody $(\mathrm{mAb})$ production constitutes the majority of the current biologics industry and antibodies are currently exclusively expressed in mammalian cells. ${ }^{1,2}$ Significant costs and timescales are associated with the manufacture of mAbs, making process efficiency a major driving force. ${ }^{3}$ For this reason, and due to the reduction in cross contamination risk and cleaning validation, there is currently a transition by the industry towards the adoption of single-use technology for the cell culture, recovery and purification stages. ${ }^{4-6}$ The technology is also being explored for the manufacture of viral vectors for gene therapy and cell therapy products.

Since the development of the first rocked bag bioreactor in 1997, other novel bioreactor designs have been developed such as orbitally shaken (SB50, Kuhner, Switzerland), two dimensionally rocked (XR20, Pall Life Sciences, Portsmouth, UK) and pneumatically driven designs (PBS, PBS Biotech, CA, US) among others ${ }^{7}$. Such a variety of designs and mixing mechanisms have further complicated the selection among different single-use products and the comparison between scales of operation.

Rigorous fluid dynamics studies in these new bioreactor designs are needed to improve understanding of the mixing characteristics, which have often resulted in improved efficiency in their utilisation. ${ }^{8,9}$ In addition, fluid dynamics has been demonstrated to have an impact on cell culture performance in terms of cell growth and antibody titre. ${ }^{10,11}$ If the type of flow present in the bioreactor is very turbulent, especially in the presence of bubbles, ${ }^{12}$ this can result 
in poorer cell yield and viability. ${ }^{13}$ Poor mixing may lead to inhomogeneity, which exposes cells to local fluctuations in $\mathrm{pH}$, dissolved oxygen, nutrient and toxin concentrations, potentially leading to higher rates of cell death. ${ }^{14}$ These can have knock-on effects on the titre and quality of the antibody products.

Rocked bag bioreactors have been adopted widely in industry. The scale limitation of commercially available rocked bags (currently the maximum working volume is $500 \mathrm{~L}$, Wave 1000/500 by GE Healthcare) means they are used mainly used as seed vessels and for clinical trial material production. Several studies have been carried out in rocked bags using a variety of organisms such as S. cerevisiae, ${ }^{15}$ Baculovirus, ${ }^{16}$ plant cells ${ }^{17}$ and E. coli $^{18}$. Rocked bag systems have also been operated in perfusion mode ${ }^{19}$ and for microcarriers suspension in stem cell expansion studies. ${ }^{20}$ Rocked bags are especially attractive because they offer efficient mixing and gas transfer and have lower shear stresses than other systems due to the lack of an impeller or dispersed gas phase. ${ }^{7}$

Only a handful of experimental and computational fluid dynamics (CFD) studies have been carried out, which aimed at characterising the flow pattern present inside a rocked bag. Velocity measurements, obtained by Oncul et al. (2009) at single points in the flow with a hot-film anemometer, were used to validate CFD simulations at 2 and $20 \mathrm{~L}$ scales. ${ }^{21}$ Oncul et al. ${ }^{21}$ described laminar conditions at rocking rate $N=15 \mathrm{rpm}$ and reported mean velocities between 0 and $0.2 \mathrm{~ms}^{-1}$ in a $2 \mathrm{~L}$ bag and between 0 and $0.6 \mathrm{~ms}^{-1}$ in a $20 \mathrm{~L} \mathrm{bag}$ over the course of one rock. The highest velocities were obtained at the centre of the bag in the main direction of motion, with low velocity gradients measured in the perpendicular direction. Further work was conducted by Kalmbach et al. (2011) ${ }^{22}$ using a hot-film based 'liquid shear stress' probe. Shear stress values of up to $0.12 \mathrm{~Pa}$ were recorded in a $2 \mathrm{~L}$ rocked bioreactor, and the authors 
postulated that such low shear stress range could be the reason for the higher cell density obtained for a microcarriers-based cultures in a similar rocked bioreactor in comparison to a stirred tank culture. The Reynolds number in rocked systems was estimated by Eibl et al. $(2009)^{23}$ using the bag length and liquid level height. Mixing time measurements were also carried out and results showed that rocking rate and fill volume have an impact on wave development and propagation and that bag geometry and scale could significantly affect the fluid flow characteristics ${ }^{23}$.

The aforementioned studies have helped elucidating some initial mixing characteristics present inside the rocked fluid motion, however the flow pattern, the mechanisms of wave propagation and the influence of the operating conditions on the velocity characteristics have not been described. In this work, for the first time, we used Particle Image Velocimetry (PIV) to measure the 2-dimensional (2-D) phase-resolved longitudinal vector field in a rocked bag system over the course of one rock at various rocking rates and fill volumes. These measurements have allowed the rigorous quantification of mean and turbulent fluid velocities and shear stress over the course of the rocking cycle at the aforementioned operating conditions. In addition, a fluid tracking methodology was developed to obtain information on the fluid surface motion and its relative position in comparison to the rocking platform.

\section{Materials and Methods}

Phase-resolved PIV was used to characterize the flow in the Perspex custom made mimic of a 2 L RM CultiBag (Sartorius Stedim, Epsom, UK). A plaster of Paris cast of the real bag system was laser scanned to digitise its shape and such information was then used to create a 1:1 scale mimic. This was manufactured by milling the top and bottom halves of the structure out of two Perspex cuboids, thus minimising refraction through curved surfaces. For further details see 
Marsh (2016) ${ }^{24}$. The rocking platform used in these experiments was fabricated in-house from welded stainless steel and powered by a PM10C 50 rpm 5.9 Nm motor (Parvalux, Dorset, UK). The platform was designed to allow the camera to be mounted on top and in order to have optical accessibility from all angles. A U-shaped through beam sensor (PMY44P, Panasonic Electric Works, NJ, USA) was blocked once per rocking cycle by a rotating spar attached to the motor. The position of this timing signal could be manually adjusted based on the inclination of the platform and provided a reference signal in correspondence to a specific inclination.

The PIV equipment used consisted of a dual head $532 \mathrm{~nm} \mathrm{Nd:YAG} \mathrm{laser,} \mathrm{a} \mathrm{NanoSense} \mathrm{MkII}$ camera with a resolution of 1260x1024 (Dantec Dynamics, Bristol, UK), a mirror and a 532 $\mathrm{nm}$ filter. With the exception of the laser, the equipment was mounted on a rocking platform as shown in the schematic diagram in Figure 1. Measurements on a vertical plane across the iii section, located $20 \mathrm{~mm}$ from the edge of the bag, were obtained using the PIV setup. Due to the symmetrical geometry of the bioreactor only half of the bag cross-section was selected as the target measurement area. The movement of the rocking platform was synchronised with the PIV acquisition system using a Texas Instruments timing system interfaced with the timing sensor, laser and camera.

The bag was filled with deionised water and seeded with $10 \mu \mathrm{m}$ silver coated hollow glass spheres (Dantec Dynamics), 500 image pairs were taken at eight phase-resolved points in the rocking cycle at the experimental conditions investigated in this work. A control study was carried out to understand the effect of image pair's number on the resulting velocity values variation and to identify a minimum number of images necessary at which to carry out all subsequent experiments. Based on this study, 500 image pairs were found to be sufficient. The 
time delay $(\Delta t)$ between two correlated images was set for each acquisition to allow the fastest particles to advance approximately one third of the interrogation cell (16x16 pixels) and varied between 1500 and $3200 \mu$ s.

For cell culture, the manufacturer's recommended rocking conditions are $\alpha=8^{\circ}$ and $\mathrm{N}=25$ rpm, where rpm indicates the number of complete rocks per minute, from $\alpha=8^{\circ}$ to $\alpha=-8^{\circ}$ and back to $\alpha=8^{\circ}$. PIV experiments were thus carried out for the platform maximum inclination up to $8^{\circ}$ above and below the horizontal. Rocking speeds $N=25,33.5$ and $42 \mathrm{rpm}$ were investigated at constant fill volume equal to $50 \%$ of the total bag volume. Fill volumes $V_{f}$ corresponding to between 30 and $60 \%$ v/v were investigated at constant rocking speed $N=$ $25 \mathrm{rpm}$. A Cartesian coordinate system $(x, y, z)$ is used which is stationary with the rocking motion, with the origin located on the rocking platform base as shown in Figure 2. Platform angle at a given inclination within a cycle $(\alpha)$ is measured in degrees above and below the horizontal, with the rotation being identified by the sign after the angle [i.e. $\alpha=+4^{\circ}$ - being $4^{\circ}$ above the centreline moving downwards]. The angle between the fluid surface and the horizontal axis of the bag is denoted by $\beta$.

While a complete dimensionless analysis is not possible in this case due to limited range of experimental conditions studied, two dimensionless numbers can be defined for the rocking system, Reynolds and Froude, and their values calculated at the three speeds investigated in this work. The characteristic velocity can be defined as the velocity at the extremity of the bag, $\alpha N L / 2$, while the half bag length, $L / 2$, can be used as the characteristic length. Reynolds and Froude numbers can therefore be defined as follows:

$$
\begin{aligned}
& R e=\alpha N L^{2} / 4 v \\
& F r=\alpha^{2} N^{2} L / 2 g
\end{aligned}
$$


Where $\alpha$ is the maximum inclination angle in radiant, $N$ is the rocking speed in rocks per second and $v$ is the fluid kinematic viscosity. Reynolds values of 1443, 1933 and 2424 were calculated for $\alpha=8$ degrees and speeds of $N=25,33.5$ and $42 \mathrm{rpm}$, respectively. Froude values of $5.4 * 10^{-5}, 9.7 * 10^{-5}$ and $1 * 10^{-4}$ were calculated for speeds of $N=25,33.5$ and $42 \mathrm{rpm}$, respectively.

A prediction of the fluid behaviour inside the rocked bag was derived from first principles on the basis that the rocking motion is responsible for the fluid motion along the length of the bag. The reference volumetric flow rate equation was estimated from a control volume analysis, where the variation of fluid in one half of the bag is equal to the amount of fluid passing through the mid-cross-section of the bag. In this analysis the fluid height at the bag mid-point was estimated assuming a stationary free surface for each angle of inclination (i.e. the free surface is always orthogonal to the gravitational force). In order to take into account the third dimension, it was assumed that the bag is a cuboid with height $H=0.05 \mathrm{~m}$, length $L=0.315 \mathrm{~m}$ and width $W=0.23 \mathrm{~m}$. Under these assumptions, the reference flow rate per unit width can be calculated by estimating the volume below the free surface at each instant and calculating its derivative in time as the bag progresses in its rotation. Equation (1) describes the flow rate derived in cases where the fluid is spread over a partial area of the bioreactor bottom:

$\frac{d V_{l}}{d t}=\frac{d \propto}{d t} \frac{L}{4} \frac{1}{(\cos (\alpha))^{2}}\left(\sqrt{2 \frac{V_{f}^{*}}{|\tan (\alpha)|}}-\frac{L}{2}\right)$

where $V_{f}^{*}$ is the fluid volume present inside the bag per unit length and $\alpha$ is the inclination angle of the platform. A corresponding reference average velocity $\overline{u_{r}}$ can be estimated by dividing the volumetric flow rate per unit width, calculated using Equation (1), by the fluid height at the bag mid-point. A full description of the derivation can be found in the Appendix. 
In order to carry out flow visualization experiments, the camera and timing system set up used for the PIV experiments was employed. The mimic was filled with $0.5 \mathrm{gL}^{-1}$ aqueous methyl blue dye at $V_{f}=50 \%$ of the bag volume and illuminated with a $650 \mathrm{~W}$ lamp. The camera was configured to capture 360 images per rocking cycle at $N=25,33.5$ and $42 \mathrm{rpm}$ (corresponding to 150,201 and $252 \mathrm{~Hz}$, respectively). Data were acquired for five rocking cycles at each speed $(N)$ and the timing system was used to start each acquisition at the same inclination during the cycle. The images obtained were then processed using a purposely-developed Matlab program to establish both the position of the bulk of the liquid and the mean angle of the free surface at 360 inclinations over one rocking cycle. A sample image obtained and schematic diagram of a representative fluid position is provided in Figure 2.

\section{Results and Discussion}

A description of the single-phase flow occurring inside the 2 L CultiBag (Sartorius) mimic is provided in this section. In the first part, PIV data were post-processed to obtain phase-resolved velocity for a reference rocking condition, namely at a rocking rate $N=25 \mathrm{rpm}$ and $50 \%$ fill volume. Phase-resolved averages were taken over a range of rocking speeds $N=25,33.5$ and $42 \mathrm{rpm}$ and fill volumes $V_{f}=30,40,50$ and $60 \%$ of the total bag volume. An analysis of the results obtained, and comparisons with previous works, is presented. Finally, the flow visualization results described in Section 3.4 were used to assess the free surface and volume variations, which were analysed at various rocking speeds.

\section{Flow pattern characterisation over one rocking cycle}

The two-dimensional (2-D) phase-resolved velocity measurements obtained in the bag vertical cross-section at $N=25 \mathrm{rpm}$ are described in detail in this section. Contour plots of the velocity magnitude, with superimposed vector field, are shown in Figure 3A-H. In Figure 3A, the platform is in a horizontal position and moving upward (see inset diagram). The fluid velocities 
are parallel to the platform base, with the fluid bulk moving from the right end of the bag at $x / L=1$ towards the centre $(x / L=0.5)$ at an average velocity magnitude of

$0.12 \mathrm{~ms}^{-1}$, estimated by averaging the velocity magnitudes over the entire section. As the platform moves upwards to $\alpha=+4^{\circ}+$ (Figure 3B), it can be observed that the fluid moves downwards along the inclined surface at a greater velocity (average velocity $0.22 \mathrm{~ms}^{-1}$ ), with velocities increasing as the fluid moves towards the centre of the bag at $x / L=0.5$.

As the platform reaches $\alpha=+8^{\circ}$ and inverts its rotation (Figure 3C), the fluid changes direction, having reached the left end side of the bag. As the platform descends to $\alpha=-4^{\circ}$ - (Figures 3DF), the fluid flows back towards $x / L=1$ at velocity magnitudes ranging from 0.12 to $0.16 \mathrm{~ms}^{-}$ ${ }^{1}$. The lowest velocity values $\left(0.026 \mathrm{~ms}^{-1)}\right.$ were achieved at $\alpha=-8^{\circ}$ (Figure 3G) as the fluid stagnates at the lower end of the bag and the platform changes direction. As the platform rotates upwards again (Figure $3 \mathrm{H}$ ) the fluid accelerates in the horizontal direction to achieve the velocity field shown in Figure 3A. Similarly to what was observed in this work, Oncul et al. $(2009)^{21}$ measured velocities using a hot-film velocity probe at various positions within a $2 \mathrm{~L}$ bag, at a rocking speed $N=15 \mathrm{rpm}$ and $7^{\circ}$ rocking angle, and reported higher velocities in the middle of the bag at $x / L=0.5$ compared to the bag edges.

The phase resolved velocity measurements also provide new insights into wave formation in the rocked bag. While the fluid predominantly flows in the direction parallel to the platform base, a few platform inclinations can be noted at which a velocity component perpendicular to the base is significant. In Figures $3 \mathrm{E}$ and $\mathrm{G}$ it can be noted that the fluid moves towards the fluid surface in correspondence to the formation of a wave on the surface itself. As the platform moves down towards the horizontal, a bulge on the liquid surface can be observed in Figure 3E. This wave may have a retarding effect on the fluid bulk movement resulting in low local 
velocity values as can be observed in Figure $3 \mathrm{E}$ at $x / L=0.67$. A similar wave formation is evident in Figure 3G at $x / \mathrm{L}=0.54$, where axial velocity components in the flow are present in correspondence to the less pronounced bulge in the fluid surface.

\section{The effect of the rocking speed on velocity characteristics and shear stress}

The two-dimensional (2-D) contour plots of the phase-resolved velocity magnitude with superimposed vector field are shown alongside contour plots of turbulent kinetic energy at various $N$ and $\alpha=-4^{\circ}$ in Figure 4 . A black line has been added to Figure 4 to visualize the inclination of the bag with respect to the horizontal and to show the extent of the fluid motion at different operating conditions. It should be noted that a stationary reference system has been used in this work, located on the platform base in correspondence to the left end of the bag. At this inclination in the rocking cycle, the platform is past the horizontal and its right hand side is moving downwards, with the fluid flowing towards the lower end of the bag $(x / \mathrm{L}=1)$.

Figures $4 \mathrm{~A}$ and $\mathrm{D}, \mathrm{B}$ and $\mathrm{E}, \mathrm{C}$ and $\mathrm{F}$ show results obtained at rocking speeds $N=25,33.5$ and $42 \mathrm{rpm}$, respectively, and at the same platform inclination with respect to the horizontal plane. A significant effect of the rocking speed can be noted both in terms of the fluid position and the velocity magnitudes across the flow field (Figures $4 \mathrm{~A}-\mathrm{C}$ ). At $N=25 \mathrm{rpm}$, the fluid direction is mainly along the platform base, with axial velocity components present only at the far right end of the bag. As the rocking speed is increased, at the same platform position, a portion of the fluid appears to be in a different location within the bag. At the highest rocking speed at which experiments were carried out, $N=42 \mathrm{rpm}$, velocities are higher than those measured at $N=25 \mathrm{rpm}$, however less fluid is present in the region comprised between $0.75<$ $x / L<1$. This could be due to the fluid moving out of phase with respect to the platform, with the majority of the fluid occupying the opposite end of the bag at this inclination in the rocking cycle (data not shown). 
Turbulent kinetic energy was calculated from the fluctuating velocity component and making the isotropic assumption as shown in Equation (2):

$k=\frac{3}{4}\left(u^{\prime 2}+w^{\prime 2}\right)$

Values of turbulent kinetic energy, $k$, below $0.02 \mathrm{~m}^{2} \mathrm{~s}^{-2}$ can be observed in Figures 4D and 4E at most locations for measurements obtained at $N=25$ and $33.3 \mathrm{rpm}$. The difference in fluid flow pattern observed at different rocking speeds generates differences in turbulent kinetic energy values measured in the bulk of the fluid. At $N=42 \mathrm{rpm}$ values of $k$ up to $0.1 \mathrm{~m}^{2} \mathrm{~s}^{-2}$ were measured, an order of magnitude higher than those observed at lower rocking speeds.

It can be hypothesized that, at the highest speeds investigated in this work, the fluid has moved out of phase with the platform and is ahead in comparison to the fluid at $N=25 \mathrm{rpm}$. The observed phenomena might generate higher turbulence levels at this rocking speed, indicated by higher turbulent kinetic energy values measured at locations close to the right hand side of the bag. Looking at Figure 4E and 4F, it suggests that the fluid has accumulated at the end of the bag and then recirculated onto itself after changing direction, which might be a cause for the observed higher turbulence levels. While the mean flow obtained at 33.5 and $42 \mathrm{rpm}$ is very similar, turbulent kinetic values up to $0.1 \mathrm{~m}^{2} \mathrm{~s}^{-2}$ can be observed at a number of locations at $N$ $=42 \mathrm{rpm}$ (Figure 4F). A flow transition from laminar to turbulent flow might be occurring at speeds between 33.5 and $42 \mathrm{rpm}$, though more experiments at intermediate speeds must be carried out to confirm this finding.

The two-dimensional (2-D) contour plots of the phase-resolved velocity magnitude with superimposed vectors field are shown alongside contour plots of shear stress at different rocking speeds and $\alpha=-8^{\circ}$ in Figure 5. It should be noted that these measurements were taken 
at the next inclination in the rocking cycle in comparison to the results shown in Figure 4A-C. At $N=25 \mathrm{rpm}$ and $\alpha=-8^{\circ}$ the fluid is moving towards the right-hand side end of the bag although axial velocity components were measured near the centre of the bag at $x / L=0.5$. The fluid surface inclination is parallel to the horizontal, as shown by the reference line in Figure 5A. As the rocking speed is increased, higher velocities were measured at locations $x / L=0.5$ 0.8, while average velocities towards the right hand side of the bag are similar to those observed at $N=25 \mathrm{rpm}$. In addition, the fluid surface inclination at $\alpha=-8^{\circ}$ deviates from being parallel to the horizontal, and a wave is formed at approximately $x / L=0.8$; this corresponds to a region of axial velocities present, at the same location, below the free surface (Figure 5B). At $N=42$ rpm the fluid has impinged on the right hand side end of the bag and is recirculating backwards towards the centre (Figure 5C).

Figures 5D-F show contour plots of one component of the Reynolds stress measured at $N=25$, 33.5 and $42 \mathrm{rpm}$, respectively. The Reynolds stress is calculated from the 2-D turbulent components of the fluid flow and has been determined using Equation (3):

$\tau_{\mathrm{uw}}=-\rho \overline{\mathrm{u}^{\prime} \mathrm{w}^{\prime}}$

From the velocity profiles obtained it is clear the flow is mostly 2-dimensional, dominated by large-scale vortices. Turbulence levels obtained at most conditions are relatively low and it can be speculated that the product u'w' is the most significant component of the shear stress tensor and therefore in this case accurate values of the shear stress can be computed from the 2-D data sets obtained with PIV. The absolute value of the shear stress was averaged over the measurement area and it was noted that such values varied significantly with increasing rocking speed. While at $N=25 \mathrm{rpm}$ the shear stress values remain below $4 \mathrm{~Pa}$ at all inclinations, values up to $15 \mathrm{~Pa}$ and $21 \mathrm{~Pa}$ were measured at $N=33.5 \mathrm{rpm}$ and at $N=42 \mathrm{rpm}$, respectively. At $\mathrm{N}$ $=33.5$ and $42 \mathrm{rpm}$ the highest shear stresses were measured at locations between $x / L=0.5-0.7$ 
and $z / H=0.25-0.30$, which is the region between the low and high velocity areas shown in the corresponding velocity plots (Figure 5B and C). Kalmbach et al. (2011) ${ }^{22}$ measured shear stresses in a $2 \mathrm{~L}$ rocked bag at $N=15 \mathrm{rpm}$ and rocking angle of $7^{\circ}$ using a hot-film probe. In their work values of shear stresses up to $0.11 \mathrm{~Pa}$ were reported based on 39 measurement points within the bag over the course of the rock.

From the results presented in Figures 3-5 it is clear that the rocked bag motion produces a characteristic flow pattern. Most importantly, the presence of a wave was observed at specific operating conditions, forming in correspondence of a characteristic velocity field and visible from the presence of a bulge in the liquid surface. It appears that the bulk flow falls further behind the platform motion as the rocking rate is increased. In order to elucidate further the observed phenomena, the results were analysed to draw additional information on the bulk fluid motion inside the bag at different $\mathrm{N}$ over the course of one rock and an effort was made to relate this motion to the movement of the platform. The horizontal velocity components were averaged across the measurement plane to obtain a single average value $(\bar{u})$ at each platform inclination in the rocking cycle. Figures 6 A-C present this value over the course of one rocking cycle for different rocking speeds. Figures 6 A-C also shows the reference velocity estimation at the relevant rocking speed, calculated as described in Section 2 from Equation (1). The volumetric flow rate provides an indication of the fluid velocity and motion caused by the free surface, which tends to remain horizontal as the base of the bag moves.

Since at low rocking speeds the platform is moving relatively slowly, gravity should be the dominant force responsible for fluid motion. The volumetric rate prediction shows a reference sinusoidal profile which is by definition in phase with the movement of the bag. Similarly at $N=25 \mathrm{rpm}$ (Figure 6A) the average velocity follows a sinusoidal profile over one rocking 
cycle. The maximum absolute average velocity is achieved at $\alpha=+4^{\circ}$ while the minimum is obtained at $\alpha= \pm 8^{\circ}$. At $N=25 \mathrm{rpm}$ the average horizontal velocity component values are very close to the profile of the reference volumetric rate, shown with a dotted line in Figure 6A-C, suggesting that the fluid motion is in phase and its behaviour predominantly determined by the gravity force drawing the fluid downwards as the bag is inclined. At a higher rocking rate of $N$ $=33.5 \mathrm{rpm}$, the average horizontal velocity follows a sinusoidal pattern, however, for most inclinations within the rocking cycle, the profile appears to be shifted towards the right by approximately $2-3^{\circ}$ in comparison to the reference profile (Figure $6 \mathrm{~B}$ ). The maximum $\bar{u}$ is greater than that obtained at $N=25 \mathrm{rpm}$ and it occurs again at $\alpha= \pm 4^{\circ}$, with the minimum velocity being present at $\alpha= \pm 8^{\circ}$. At $N=42 \mathrm{rpm}$ (Figure 6C) a sinusoidal profile is observed which, similarly to what has been observed at $N=33.5 \mathrm{rpm}$, appears to be shifted by $3-4^{\circ}$ in comparison to the rocking cycle observed at $N=25 \mathrm{rpm}$ and with respect to the reference curve.

Fluid velocities measured by Oncul et al. ${ }^{21}$ in a $2 \mathrm{~L}$ rocked bag at $N=15 \mathrm{rpm}$ showed a similar trend, with maximum average velocities of $0.1-0.2 \mathrm{~ms}^{-1}$ reported at various locations in the bag at an inclination of $\alpha=0^{\circ}$, and minimum average velocity values around or less than 0.02 $\mathrm{ms}^{-2}$ obtained at $\alpha=8^{\circ}$. The volumetric flow rate estimation described by Equation (1) would predict values of approximately $0.12 \mathrm{~ms}^{-1}$ at a rocking speed $N=15 \mathrm{rpm}$. These results provide an indication of the fluid location with respect to the bag movement and, under the assumptions made in this work, the fluid moves out of phase with the platform as the rocking speed is increased. The phenomena, combined with the presence of higher turbulence levels observed at these rocking speeds, might have implications for cell culture of suspended and adherent cell lines in this bioreactor geometry.

\section{The effect of fill volume on the velocity field}


Fluid working volume is an important variable that has not been frequently investigated in rocked bag bioreactors. Fill volume varies significantly during cell culture, however, depending on sampling and feeding procedures, thus having an impact upon power input per unit volume. In this work, phase-resolved data were obtained at fluid working volumes of 30, 40,50 and $60 \%$ of the total bag volume.

Figure 7 shows the contour plots of the phase-resolved velocity magnitude with superimposed vectors field for each of the fluid working volumes investigated at two consecutive measurement angles, $\alpha=0^{\circ}-$ and $\alpha=-4^{\circ}-$ at $N=25 \mathrm{rpm}$. At this point in the rocking cycle, the fluid is flowing into one end of the bag. Interestingly a significant effect of the liquid volume is noted on the velocity magnitude, which is in general higher at lower fill volumes. Average horizontal velocities, determined by taking the average over the measurement area for all inclinations within one cycle, were found to be $0.116,0.105,0.092$, and $0.076 \mathrm{~ms}^{-1}$ for fill volumes of 30, 40, 50 and $60 \%$ respectively. At $\alpha=-4^{\circ}$ - a wave is formed on the liquid surface as the liquid impinges on the end of the bag and inverts direction; a phenomenon that is visible at all fill volumes in correspondence to different horizontal locations (Figures E-H).

Phase-resolved horizontal velocity components were averaged over the measurement area $(\bar{u})$ at different fill volumes and $N=25 \mathrm{rpm}$ and the resulting velocity values are presented in Figure 8 for all inclinations within one rocking cycle. All profiles exhibit a sinusoidal trend, similar to what was observed at different rocking speeds (Figure 6). Lower fill volumes resulted in higher average $\bar{u}$, with maximum values $\bar{u}=0.11 \mathrm{~ms}^{-1}$ and $0.16 \mathrm{~ms}^{-1}$ obtained at $60 \%$ and $30 \%$ fill volume, respectively. It is noteworthy that at $60 \%$ fill volume the maximum velocity is achieved in correspondence to an inclination $\alpha=0^{\circ}$, while at decreasing fill volumes the maxima is achieved at $\alpha=-4^{\circ}$. As it was discussed in relation to rocking speed, the fill volume 
has an effect on the average fluid flow pattern and the fluid moves out of the phase with the platform movement as the fill volume is decreased.

\section{Fluid flow visualization}

Flow visualisation experiments were performed over the full bag cross-section to validate the PIV results and improve understanding of the fluid motion over one rocking cycle. Image acquisition was synchronised to the bag angular position by means of an encoder, while the high frame rate, 360 time steps over one rocking cycle, allowed an accurate resolution of the shape of the free surface for each phase angle. An average estimation of the free surface inclination with respect to the angular position of the bag, $\beta$, was obtained by linear interpolation, while to best capture the overall position of the fluid with the bag the location of the 2-D centre of mass (centroid) was determined at each inclination.

Figure 9A shows the variation of the fluid surface angle with platform inclination for one cycle at different rocking speeds. It is clear that the fluid surface changes inclination with respect to the horizontal quite suddenly and at the same platform inclination for all rocking speeds. However, the fluid surface angle $(\beta)$ is changing more significantly at higher rocking speeds. In addition, as the rocking speed is increased, the $\beta$ profile shifts towards the right hand side, indicating that the maximum fluid inclinations are achieved at a different rocking angle and later in the rocking cycle. Maximum $\beta$ values obtained were $8^{\circ}$ at $N=25 \mathrm{rpm}\left(\right.$ at $\left.\alpha=+8^{\circ}\right)$ and $14^{\circ}$ at $N=42 \mathrm{rpm}$ (at approximately $\alpha=+6^{\circ}$ ).

Integration of the absolute value of $\beta$ over a complete rocking cycle could provide an indication of the total extent to which the fluid surface angle differs from the horizontal. Figure 9C shows

the results of this integration for different rocking speeds. The average surface angle $\bar{\beta}$ increases with respect to the rocking speed, with the fluid moving increasingly out of phase at 
higher rocking speeds. Fluid surface inclination data has been obtained by Kalmbach et al. $(2011)^{22}$ using a capacitance probe. In their work data were reported for a $2 \mathrm{~L}$ rocked bag at $N$ $=15 \mathrm{rpm}$ and $7^{\circ}$ rocking angle and used to integrate the surface angle profile. A value of $1.7^{\circ}$ was obtained by Kalmbach et al. (2011) $)^{22}$, which corresponds to the inclination obtained at a rocking speed of approximately $20 \mathrm{rpm}$ based on the results shown in Figure 9C. This comparison with $\mathrm{Kalmbach}^{22}$ work, even though based on one experimental data point, suggests that the linear range observed between $N=25$ and $42 \mathrm{rpm}$ may not extend at speeds below $N=25 \mathrm{rpm}$ and a different mathematical relationship might exist between $\bar{\beta}$ and $N$ in this range.

The normalised horizontal position of the fluid centroid $\left(x_{C} / L\right)$ with respect to the rocking platform angle is presented in Figure 9B at different rocking speeds. In Figure 9B the centroid location obtained at the reference average velocity, estimated using Equation (1), is also indicated by the solid grey line. The normalised centroid position $\left(x_{C} / L\right)$ follows a sinusoidal profile, with the fluid position changing similarly for all speeds investigated. The results presented confirm that at $N=25 \mathrm{rpm}$ the bulk fluid position corresponds to the fluid movement obtained with the analysis leading to Equation (1), with a time delay being evident and more prominent as $N$ is increased. The observed fluid behaviour might have implications for the selection of operating conditions of different biological applications for which the rocked bioreactor is often used. As an example, these bioreactors are increasingly used for process development studies on the suspension of microcarriers-type particles and adherent cell cultivation and the movement of the fluid, whether in phase or not with the movement of the platform, might affect the overall suspension characteristics and the extent of carrier-to-carrier collisions. 
To contextualise the findings obtained on the flow characteristics in rocked bioreactors at different operating conditions, it is important to make a comparison with the velocity values reported for a traditional stirred reactor at a similar scale. In order to make a realistic comparison between the two bioreactor types, dimensionless quantities should have been used representing the flow regime under which the experiments were obtained. As such analysis is not yet available for rocked bioreactors, it was deemed useful to compare absolute values in order to provide an indication of how the characteristics differ between the two configurations.

Figure 10 shows a summary of the average and maximum velocity magnitudes and turbulent kinetic energy values measured at different rocking speeds in this work, alongside values extrapolated from the work of Kalmbach et al. $(2011)^{22}$ for a rocked bioreactor at $N=15 \mathrm{rpm}$. The average value was determined by calculating the average of each characteristic across the measurement section for all inclinations, while the maximum value represents the highest characteristic magnitude measured at all inclinations for a specific rocking speed. As expected, both maximum and average values were found to increase with $N$, with average turbulent kinetic energy increasing over the $N$ range from $0.0019 \mathrm{~m}^{2} \mathrm{~s}^{-2}$ at $N=25 \mathrm{rpm}$ to $0.019 \mathrm{~m}^{2} \mathrm{~s}^{-2}$ at $N=42 \mathrm{rpm}$. Comparison of these values to those measured in a laboratory scale, downward pumping pitched-blade turbine $\left(V_{\text {tip }}=1.06 \mathrm{~ms}^{-1}\right)^{25}$ showed close agreement with the values obtained using a rocking speed $N=42 \mathrm{rpm}$, the only exception being the greater range of turbulent kinetic energy values measured at $N=33.5$ and $N=42 \mathrm{rpm}$. While a dimensional analysis is necessary to be able to compare the flow regimes and the extent of turbulent levels present in the two bioreactors, it is notable that the turbulent kinetic energy values in the rocked bioreactor might be higher than those commonly found in stirred configurations, confirming the importance of selecting operating conditions based on fluid dynamics characteristics and 
engineering findings. It is noteworthy that comparison with the work of Kalmbach et al. $(2011)^{22}$ show promise and a very good fit was found with the data obtained in this work.

\section{Concluding remarks}

The experimental fluid dynamic investigation carried out in this work has helped elucidate the whole flow field present within a rocked bag bioreactor for the first time. Advanced manufacturing methods (3D imaging and $\mathrm{CNC}$ machining) allowed the development of an exact bag replica of a $2 \mathrm{~L}$ commercial bioreactor and the construction of a customised rocking platform. Fluid dynamic characteristics like velocity magnitude, shear stress and turbulent kinetic energy were obtained for a range of liquid fill volumes and rocking speeds at different inclinations within the rocking cycle.

The results obtained revealed the flow pattern present at different operating conditions, showing the presence of a wave on the free surface which only appeared when there was a significant velocity component perpendicular to the base. Higher turbulent kinetic energy values were found to be present at the highest rocking speed investigated, $N=42 \mathrm{rpm}$, in correspondence to the fluid moving out of phase with the platform motion. Results show that the bulk flow falls further behind the platform motion as the rocking rate is increased. A prediction of the fluid behaviour inside the bag was derived from first principles using a control volume approach on the basis that the rocking motion is responsible for the fluid motion along the length of the bag and the free surface is always orthogonal to the gravitational force. The resulting average velocity has provided a useful comparison with the velocity variation profiles within one rock obtained at different rocking speeds and has highlighted how the fluid motion changes with operating conditions. The impact of liquid fill volume on velocity characteristics was also ascertained, with higher fill volumes resulting in lower average velocities. 
In this work experiments were conducted between $N=25$ and $N=42 \mathrm{rpm}$ to consider the full range of working speeds commonly used for bioprocess development suggested by the equipment manufacturer. From a fluid dynamic point of view it would be interesting to extend the speed range at $N<25 \mathrm{rpm}$. In fact, at such lower speeds, the liquid surface would be flat and quasi-static flow below the free surface could be studied at different inclinations. In addition, a flow transition from laminar to turbulent flow might occur at around $N=42 \mathrm{rpm}$ and additional experiments in a wider range of speeds are crucial to elucidate this phenomenon. Findings obtained in this work have demonstrated a strong variation of $\beta$ at large values of $\alpha$. The wave formation and fluid recirculation inside the bag might be described by a slosh dynamics problem where the dynamics of the liquid is affected and significantly altered by the interactions with the container. A numerical analysis based on computational fluid dynamics and finite element methods could be used to solve the fluid-structure interaction problem in the presence of a flexible structure (bag).

Overall this work has provided a rigorous insight into the rocking motion and the extensive data set will be crucial for the development of an analytical model, the selection of conditions depending on process requirements as well as for the validation of computational codes. In addition, the data generated can be used as a base to develop generic equations, able to relate the flow characteristics present in the rocking bioreactor to other commonly used configurations and support technology translation from conventional to/from single-use technologies and bioprocess scale-up.

\section{Notation}

Fr Froude number [-]

$H \quad$ height of the bag bioreactor [m]

$k \quad$ turbulent kinetic energy $\left[\mathrm{m}^{2} \mathrm{~s}^{-2}\right]$ 
$L \quad$ length of the bag bioreactor [m]

$N \quad$ rocking rate [rocks per minute, rpm; rocks per second, rps, in Equation (4) and (5)]

Re Reynolds number [-]

$t \quad$ time $[\mathrm{s}]$

$x, y, z$ Cartesian reference coordinates [-]

$x_{c} \quad \mathrm{x}$ coordinate of the centroid location [m]

$u^{\prime} \quad$ horizontal fluctuating velocity component $\left[\mathrm{ms}^{-1}\right]$

$\bar{u} \quad$ horizontal velocity average $\left[\mathrm{ms}^{-1}\right]$

$\overline{u_{r}} \quad$ reference average velocity $\left[\mathrm{ms}^{-1}\right]$

$\dot{V} \quad$ reference volumetric flow rate per unit width $\left[\mathrm{m}^{2} \mathrm{~s}^{-1}\right]$

$V_{f} \quad$ fluid volume $\left[\mathrm{m}^{3}\right]$

$V_{f}^{*} \quad$ fluid volume per unit width $\left[\mathrm{m}^{2}\right]$

$V_{\text {tip }} \quad$ impeller tip speed $\left[\mathrm{ms}^{-1}\right]$

$w^{\prime} \quad$ axial fluctuating velocity component $\left[\mathrm{ms}^{-1}\right]$

W width of the bag bioreactor [m]

\section{Greek Symbols}

$\alpha \quad$ rocking platform inclination angle [ ${ }^{\circ}$, radiant in Equation (4) and (5)]

$\beta \quad$ free surface inclination angle $\left[{ }^{\circ}\right]$

$v \quad$ kinematic viscosity $\left[\mathrm{m}^{2} \mathrm{~s}^{-1}\right]$

$\rho \quad$ fluid density $\left[\mathrm{kgm}^{-3}\right]$

$\tau_{u v} \quad$ Reynolds stress [Pa] 


\section{Literature Cited}

1 Rodrigues ME, Costa AR, Henriques M, Azeredo J, Oliveira R. Technological Progresses in Monoclonal Antibody Production Systems. Biotechnol Prog. 2009;26;2:332-351.

2 Agarwaal S. What's fueling the biotech engine - 2012 to 2013. Nature Biotechnol. 2014;32;1:32-39.

3 Stock R. A Significant and Growing Market: Single Use Technology in the Biopharmaceutical Industry. Am. Pharma. Rev. 2010.

4 Rao G, Moreira A, Brorson K. Disposable bioprocessing: The future has arrived. Biotechnol. Bioeng. $2009 ; 102 ; 2: 348-356$.

5 Novais JL, Titchener-Hooker NJ, Hoare M. Economic comparison between conventional and disposables-based technology for the production of biopharmaceuticals. Biotechnol. Bioeng. $2001 ; 75 ; 2: 143-153$.

6 Lim JAC, Sinclair A. Process economy of disposable manufacturing: Process models to minimize upfront investment. Am. Pharm. Rev. 2007;10:114-121.

7 Singh V. Disposable bioreactor for cell culture using wave-induced agitation. Cytotechnol. 1999;30;1-3:149-158.

8 Barrett TA, Wu A, Zhang H, Levy MS, Lye GJ. Microwell Engineering Characterization for Mammalian Cell Culture Process Development. Biotechnol. Bioeng. 2010;105;2:260-275.

9 Micheletti M, Barrett T, Doig SD, Baganz F, Levy MS, Woodley JM, Lye GJ. Fluid mixing in shaken bioreactors: Implications for scale-up predictions from microlitre-scale microbial and mammalian cell cultures. Chem. Eng. Sci. 2006;61:2939-2949.

10 Schmalzriedt S, Jenne M, Mauch K, Reuss M. Integration of Physiology and Fluid Dynamics. Adv. Biochem. Eng./Biotechnol. 2003;80:19-68.

11 Marks DM. Equipment design considerations for large scale cell culture. Cytotechnology. 2003;42:21-33.

12 Chisti Y. Animal-cell damage in sparged bioreactors. Trends in Biotechnol. 2000;18;10:420-432. 
13 Oh SKW, Nienow AW, Al-Rubeai M, Emery AN. Further studies of the culture of mouse hybridomas in an agitated bioreactor with and without continuous sparging. J. Biotechnology. $1992 ; 22: 245-270$

14 Nienow AW. Reactor engineering in large scale animal cell culture. Cytotechnology. 2006;50:9-33. 15 Mikola M, Seto J, Amanullah A. Evaluation of a novel Wave Bioreactor cellbag for aerobic yeast cultivation. Bioprocess Biosyst. Eng. 2007;30:231-241.

16 Weber W, Weber E, Geisse S, Memmert K. Optimisation of protein expression and establishment of the Wave Bioreactor for Baculovirus/insect cell culture. Cytotechnology. 2002;38:77-85.

17 Eibl R, Werner S, Eibl D. Disposable bioreactors for plant liquid cultures at Litre-scale. Eng. Life Sci. 2009;9;3:156-164.

18 Mahajan E, Matthews T, Hamilton R, Laird M. Use of Disposable Reactors to Generate Inoculum Cultures for E. coli Production Fermentations. Biotechnol. Prog. 2010;26;2:332-351.

19 Tang YJ, Ohashi R, Hamel JFP. Perfusion culture of Hybridoma cells for Hyperproduction pf $\operatorname{IgG}_{2 \mathrm{~A}}$ Monoclonal Antibody in a Wave Bioreactor-Perfusion Culture System. Biotechnol. Prog. 2007;23:255264.

20 Genzel Y, Behrendt I, König S, Sann H, Reichl U. Metabolism of MDCK cells during cell growth and influenza virus production in large-scale microcarrier culture. Vaccine. 2004;22(17-18):22022208.

21 Öncül AA, Kalmbach A, Genzel Y, Reichl U, Thévenin D. Characterization of Flow Conditions in 2 L and 20 L Wave Bioreactors Using Computational Fluid Dynamics. Biotechnol. Prog. 2010;26;1:101-110.

22 Kalmbach A, Bordás R, Öncül AA, Thévenin D, Genzel Y, Reichl U. Experimental Characterization of Flow Conditions in 2- and 20-L Bioreactors with Wave-Induced Motion. Biotechnol. Prog. 2011;27;2:402-409.

23 Eibl R, Werner S, Eibl D. Bag Bioreactor Based on Wave-Induced Motion: Characteristics and Applications. Adv. Biochem. Engin./Biuotechnol. 2009;115:55-87.

24 Marsh DTJ Engineering Characterisation of a rocked bag bioreactor for improved process development and scale-up. University College London. EngD Thesis (2016). 
25 Gabriele A, Nienow AW, Simmons MJH. Use of angle resolved PIV to estimate local specific energy dissipation rates for up- and down-pumping pitched blade agitators in a stirred tank. Chem. Eng. Sci. 2009;64;1:126-143. 


\section{List of figure captions}

Fig.1 Schematic configuration of the experimental set up and target measurement area (i-ii plane). A top view; B,C side view (distances are reported in $\mathrm{mm}$ ).

Fig.2 Flow visualisation in the structural mimic: A Image of the fluid flow position in the mimic at a fixed angle, determination of the outer bound of the liquid (red line) and estimation of the liquid surface inclination (green line). B Schematic diagram of a representative bag and fluid position.

Fig.3 Phase-resolved velocity vector fields and contour plots of the velocity magnitude in the bag cross-section at $N=25 \mathrm{rpm}$ for a complete rocking cycle: A $\alpha=0^{\circ}+$; B $\alpha=+4^{\circ}+$; $\mathrm{C} \alpha=$ $+8^{\mathrm{o}}$; D $\alpha=+4^{\mathrm{o}}-$; E $\alpha=0^{\mathrm{o}}-; \mathrm{F} \alpha=-4^{\mathrm{o}}-; \mathrm{G} \alpha=-8^{\mathrm{o}} ; \mathrm{H} \alpha=-4^{\mathrm{o}}+$. Here '+' refers to ascending motion and '-' to descending motion. White area in middle of velocity fields indicates area where a bolt through the structural mimic obscured the laser.

Fig.4 Phase-resolved velocity vector fields and contour plots of the velocity magnitude (A-C) and turbulent kinetic energy $(\mathrm{D}-\mathrm{F})$ at $\alpha=-4^{\circ}$ for rocking rates: $N=25 \mathrm{rpm}(\mathrm{A}$ and $\mathrm{D}) ; N=$ $33.5 \mathrm{rpm}(\mathrm{B}$ and $\mathrm{E}) ; N=42 \mathrm{rpm}(\mathrm{C}$ and $\mathrm{F})$.

Fig.5 Phase-resolved velocity vector fields and contour plots of the velocity magnitude (A-C) and shear stress (D-F) at $\alpha=-8^{\circ}$, for rocking rates $N=25 \mathrm{rpm}(\mathrm{A}$ and $\mathrm{D}) ; N=33.5 \mathrm{rpm}(\mathrm{B}$ and $\mathrm{E}) ; N=42 \mathrm{rpm}(\mathrm{C}$ and $\mathrm{F})$.

Fig.6 A-C Average velocity profiles at different inclinations within a rocking cycle; PIV data

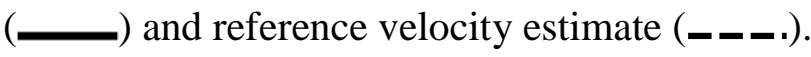

Fig.7 Phase-resolved velocity vector fields and contour plots of the velocity magnitude at $N=$ $25 \mathrm{rpm}$ at $\alpha=0^{\circ}(\mathrm{A}-\mathrm{D})$ and $\alpha=-4^{\circ}(\mathrm{E}-\mathrm{H})$ for fill volumes of $30 \%$ of the total bag volume (A and $\mathrm{E}) ; 40 \%$ (B and $\mathrm{F}), 50 \%(\mathrm{C}$ and $\mathrm{G}) ; 60 \%(\mathrm{D}$ and $\mathrm{H})$.

Fig.8 Average velocity profile at different inclinations within one cycle for different fill

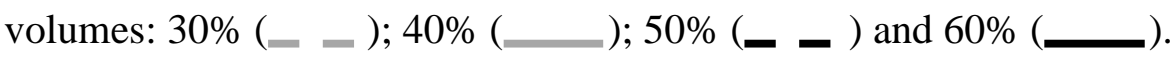


Fig.9 Fluid surface angle (A) and centroid location (B) at different inclinations within one rocking cycle at 25rpm (—), 33.5rpm (_- - .), 42rpm (.......) and reference velocity estimate (_ ) . C Magnitude of the average fluid surface angle $(\beta)$ calculated within one rocking cycle. Solid line fitted by linear regression $\left(\mathrm{R}^{2}=0.99\right)$. Error bars represent one standard deviation about the mean $(n=3)$.

Fig.10 Comparison of average $(\bullet)$ and maximum $(\times)$ velocities and turbulent kinetic energy at different rocking rates. Grey symbols represent data extrapolated from the work of Kalmbach et al., 2011. Dashed and dotted lines represent average and maximum values, respectively, for a 2.7 L stirred tank bioreactor reported by Gabriele et al., 2009. 

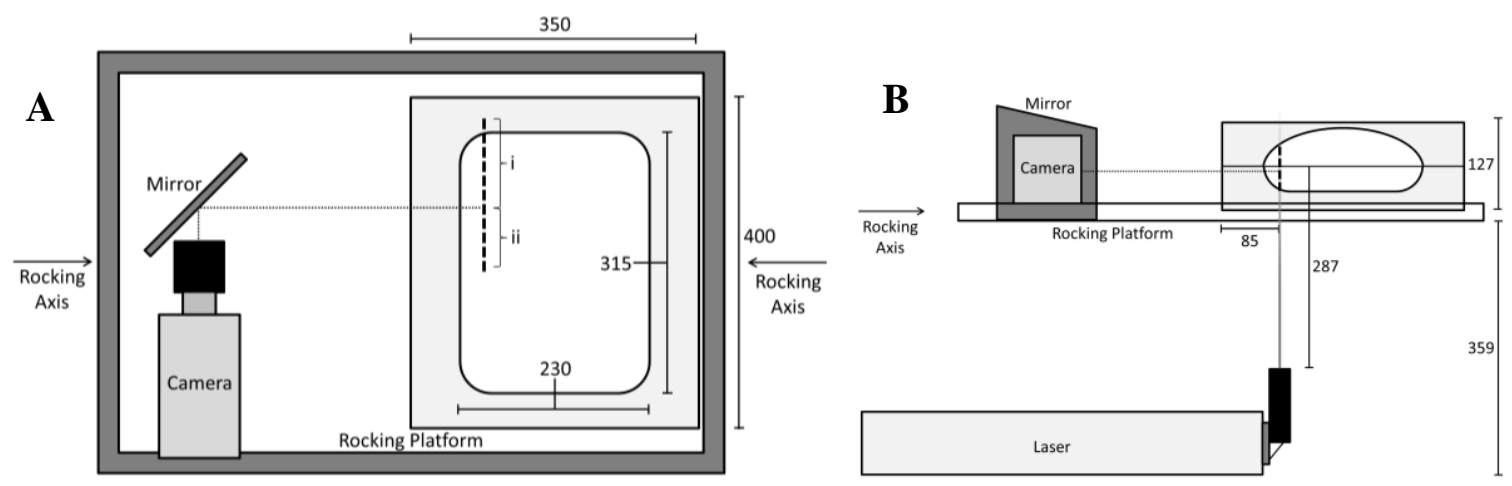

C

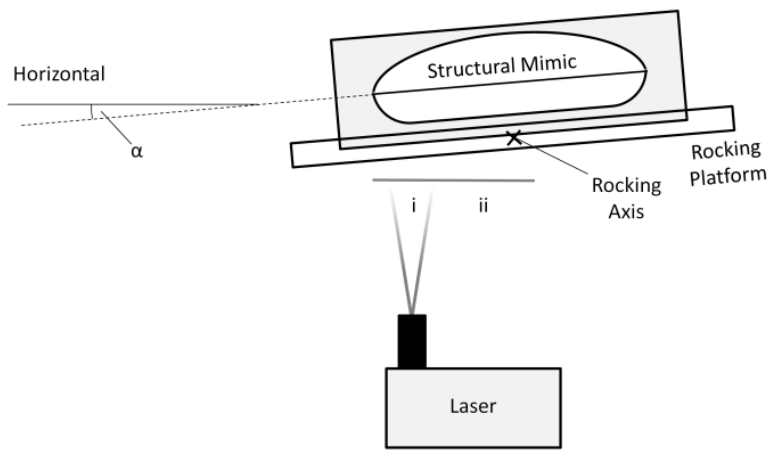

Fig.1 Schematic configuration of the experimental set up and target measurement area (i-ii plane). A top view; B,C side view (distances are reported in $\mathrm{mm}$ ). 
A
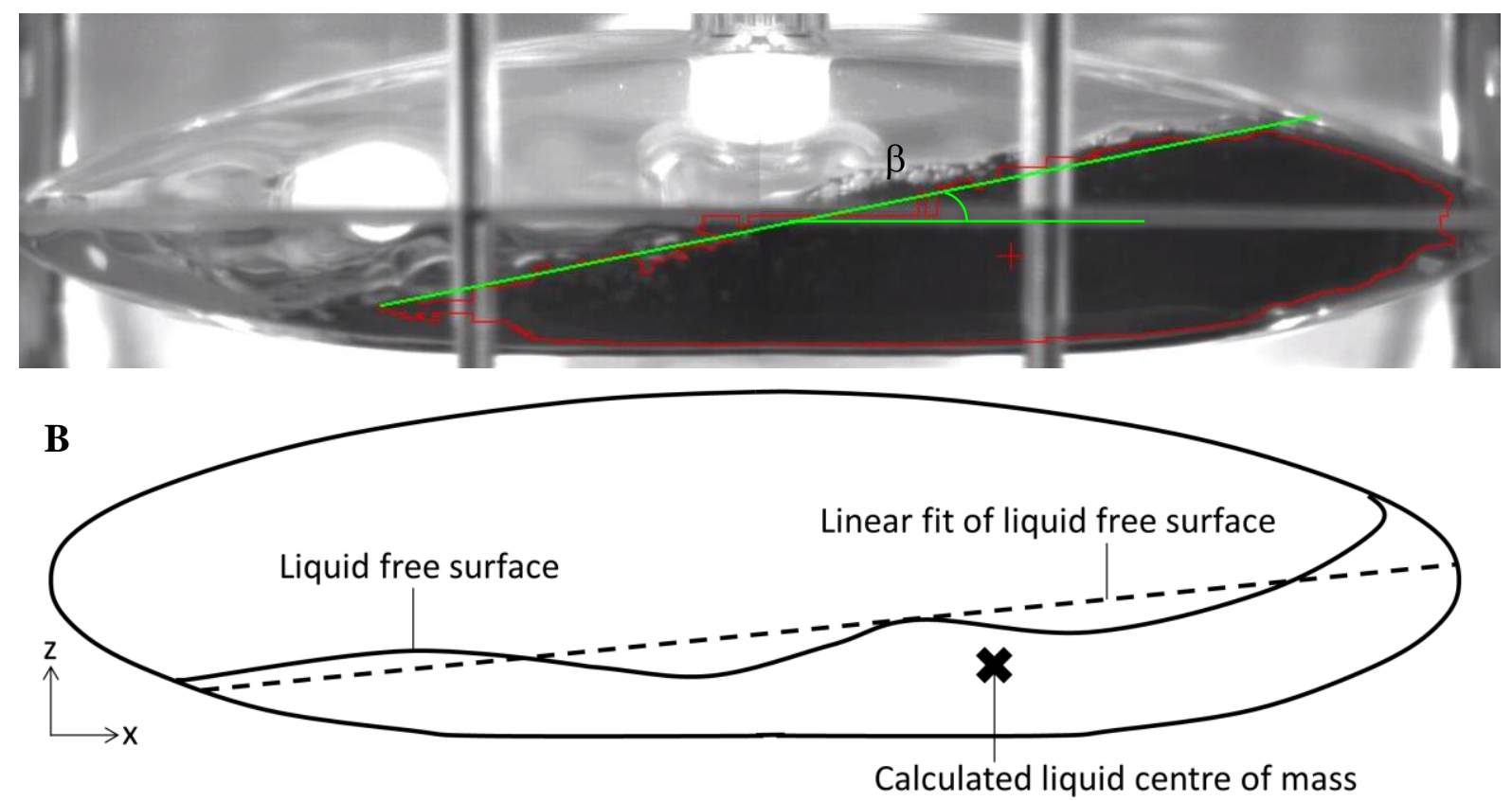

Fig.2 Flow visualisation in the structural mimic: A Image of the fluid flow position in the mimic at a fixed angle, determination of the outer bound of the liquid (red line) and estimation of the liquid surface inclination (green line). B Schematic diagram of a representative bag and fluid position. 

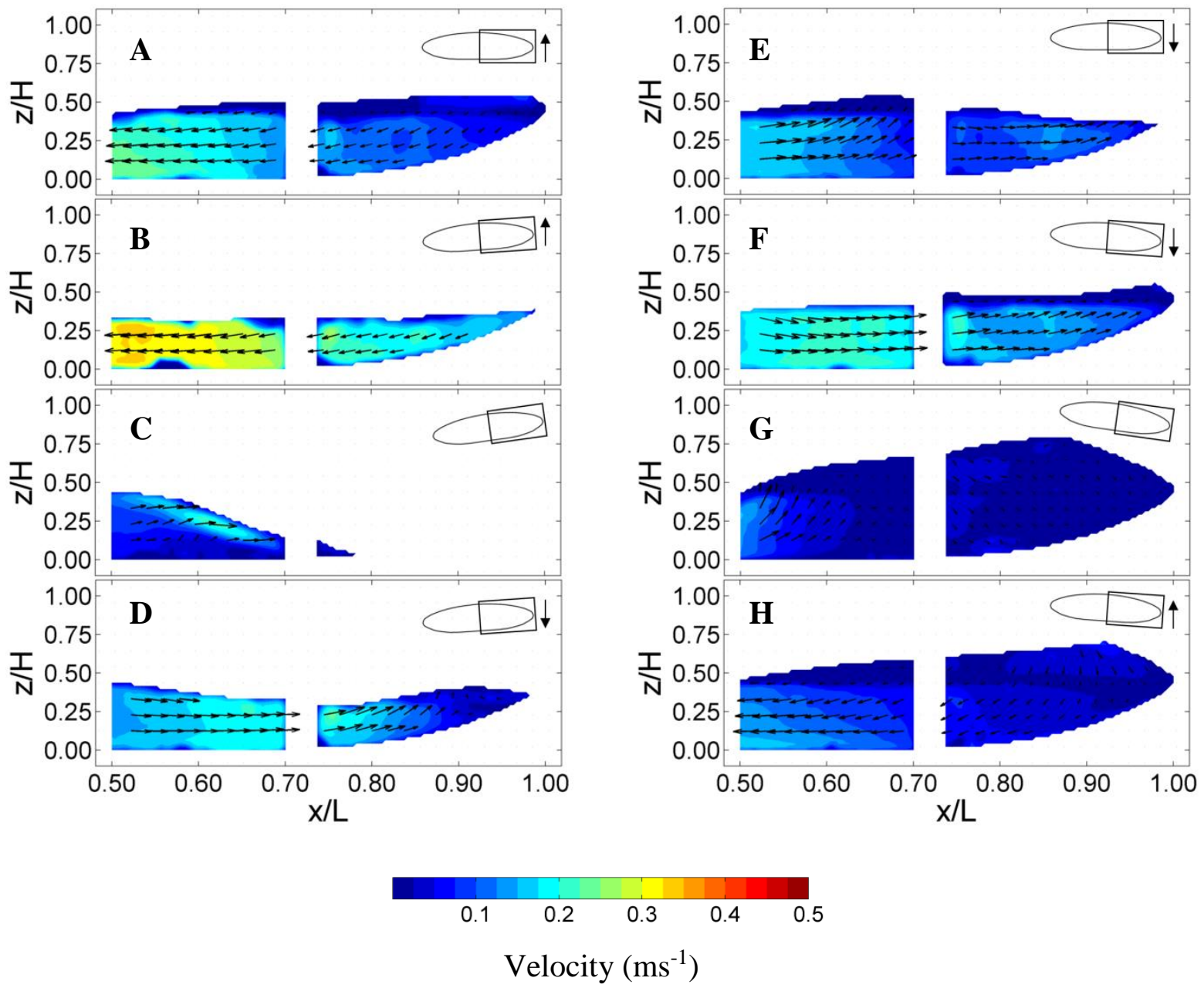

Fig.3 Phase-resolved velocity vector fields and contour plots of the velocity magnitude in the bag cross-section at $N=25 \mathrm{rpm}$ for a complete rocking cycle: A $\alpha=0^{\circ}+; \mathrm{B} \alpha=+4^{\circ}+$; $\mathrm{C} \alpha=$ $+8^{\mathrm{o}}$; $\mathrm{D} \alpha=+4^{\mathrm{o}}-$; E $\alpha=0^{\circ}-$; F $\alpha=-4^{\mathrm{o}}-$; G $\alpha=-8^{\mathrm{o}}$; H $\alpha=-4^{\mathrm{o}}+$. Here '+' refers to ascending motion and '-' to descending motion. White area in middle of velocity fields indicates area where a bolt through the structural mimic obscured the laser. 

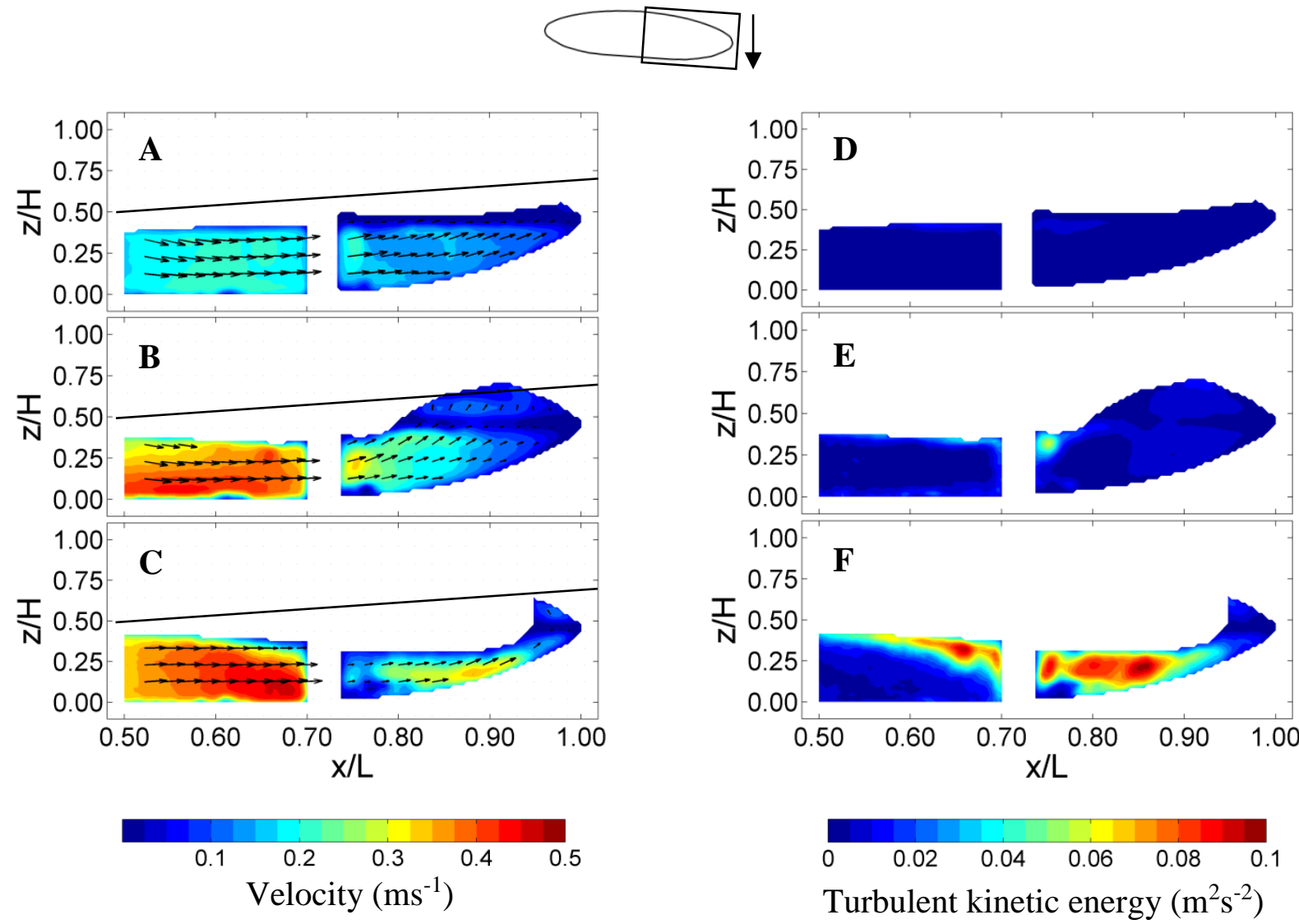

Fig.4 Phase-resolved velocity vector fields and contour plots of the velocity magnitude (A-C) and turbulent kinetic energy (D-F) at $\alpha=-4^{\circ}$ for rocking rates: $N=25 \mathrm{rpm}(\mathrm{A}$ and $\mathrm{D}) ; N=$ $33.5 \mathrm{rpm}(\mathrm{B}$ and $\mathrm{E}) ; N=42 \mathrm{rpm}(\mathrm{C}$ and $\mathrm{F})$. 

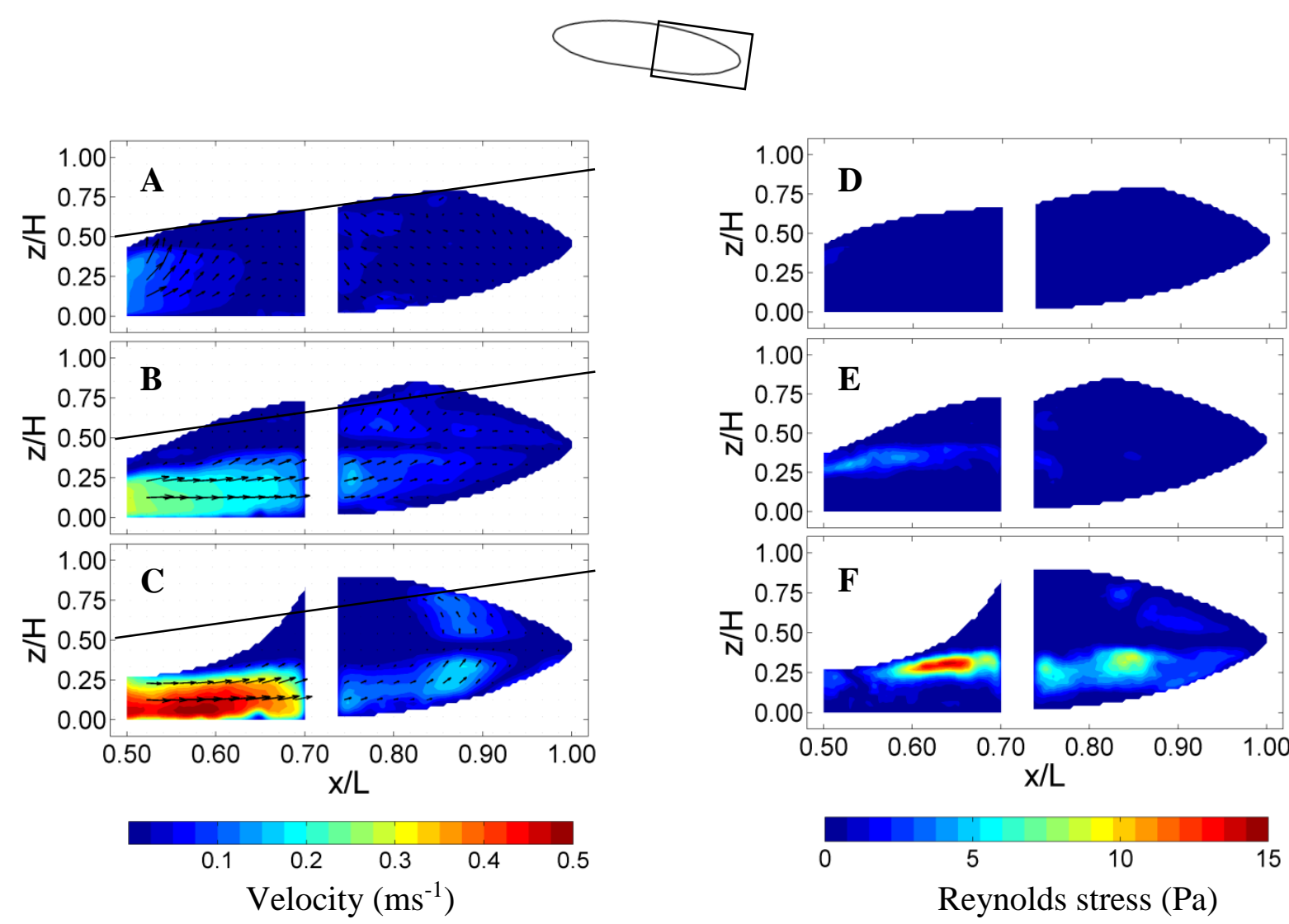

Fig.5 Phase-resolved velocity vector fields and contour plots of the velocity magnitude (A-C) and shear stress (D-F) at $\alpha=-8^{\circ}$, for rocking rates $N=25 \mathrm{rpm}(\mathrm{A}$ and $\mathrm{D}) ; N=33.5 \mathrm{rpm}(\mathrm{B}$ and $\mathrm{E}) ; N=42 \mathrm{rpm}(\mathrm{C}$ and $\mathrm{F})$. 

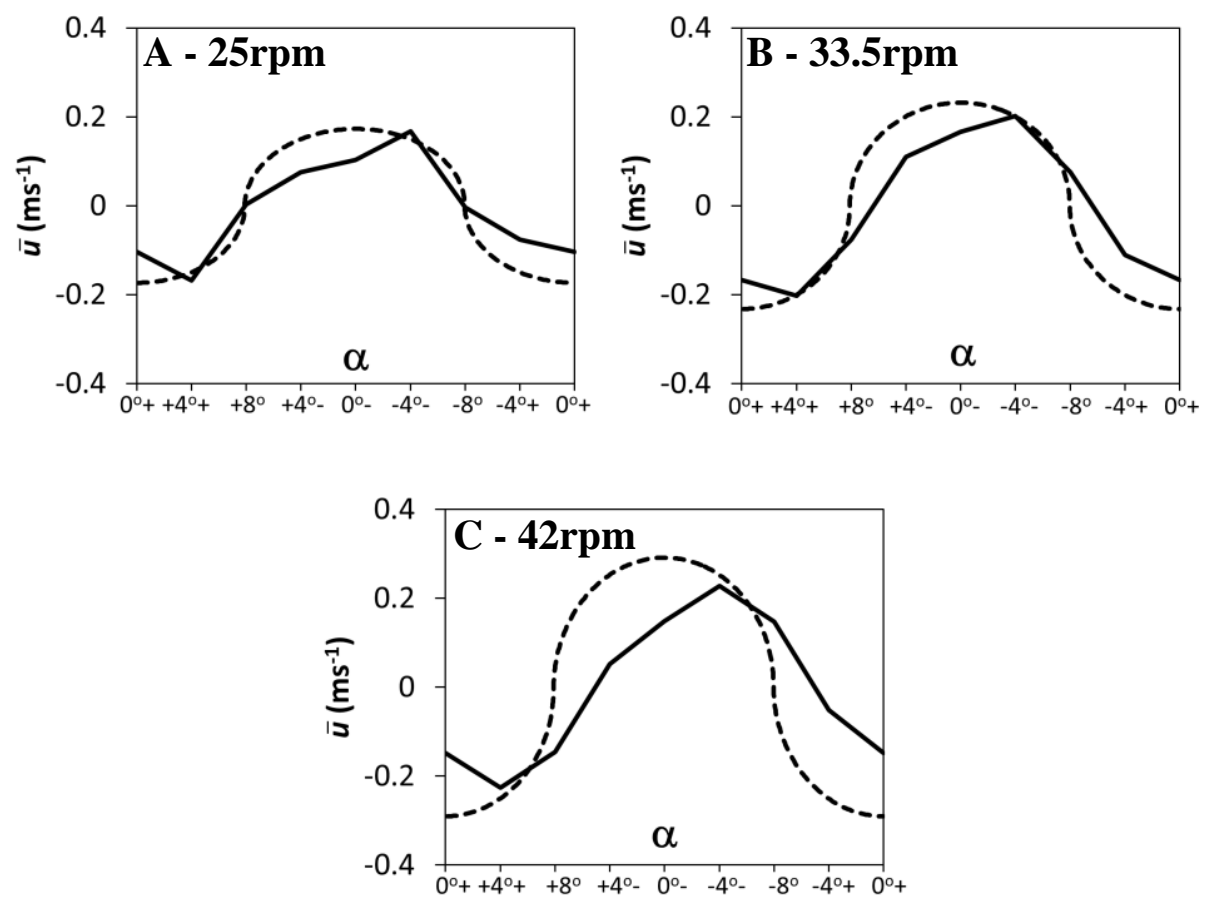

Fig.6 A-C Average velocity profiles at different inclinations within a rocking cycle; PIV data (—) and reference velocity estimate (_- - .). 

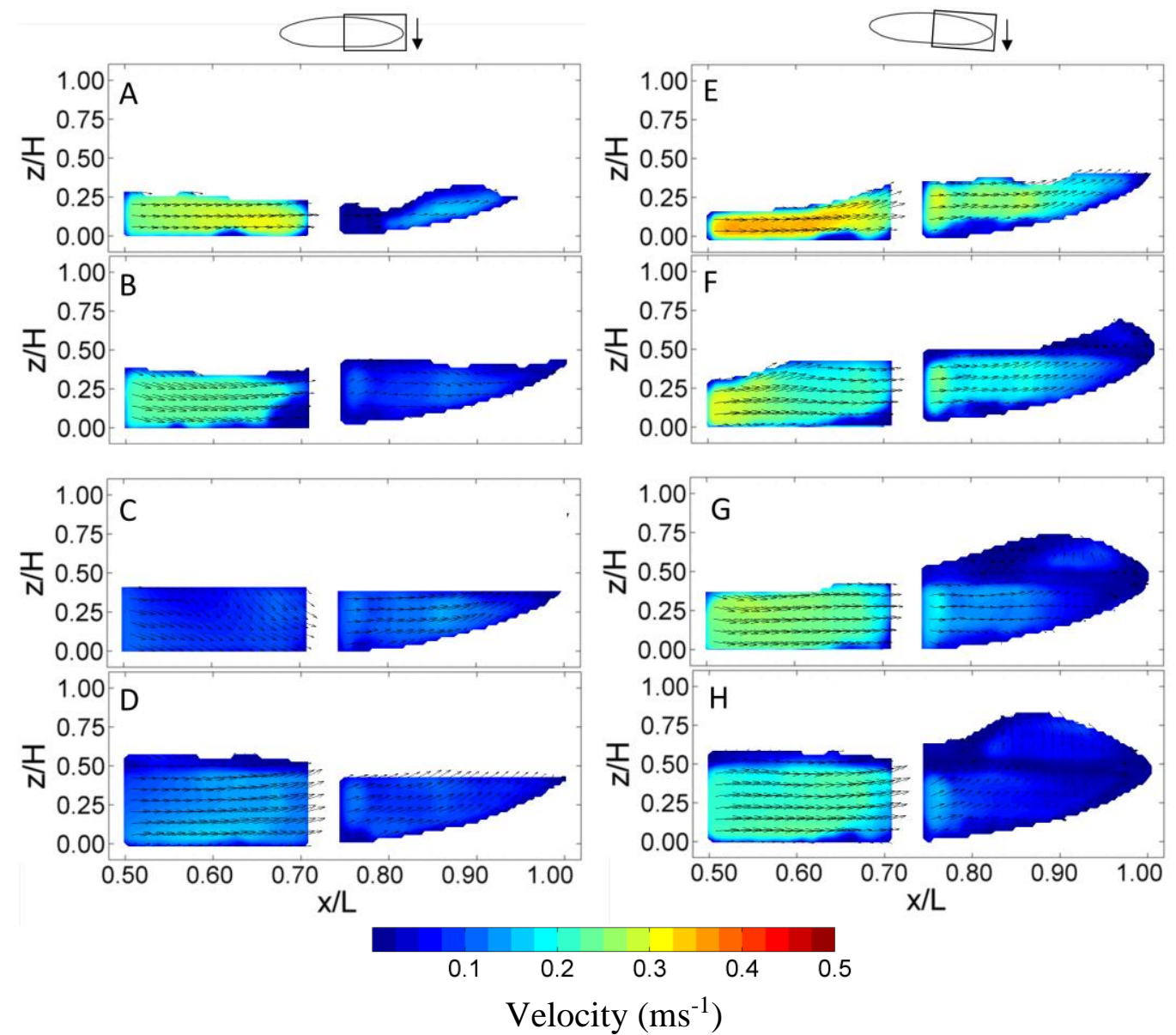

Fig.7 Phase-resolved velocity vector fields and contour plots of the velocity magnitude at $N=$ $25 \mathrm{rpm}$ at $\alpha=0^{\circ}(\mathrm{A}-\mathrm{D})$ and $\alpha=-4^{\circ}(\mathrm{E}-\mathrm{H})$ for fill volumes of $30 \%$ of the total bag volume (A and $\mathrm{E}) ; 40 \%$ (B and F), 50\% (C and $\mathrm{G}) ; 60 \%$ (D and $\mathrm{H}$ ). 


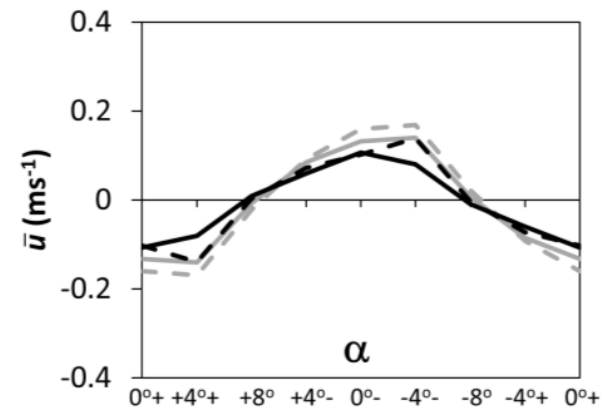

Fig.8 Average velocity profile at different inclinations within one cycle for different fill

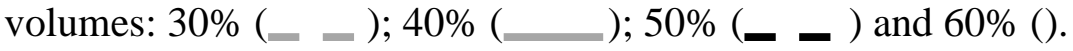



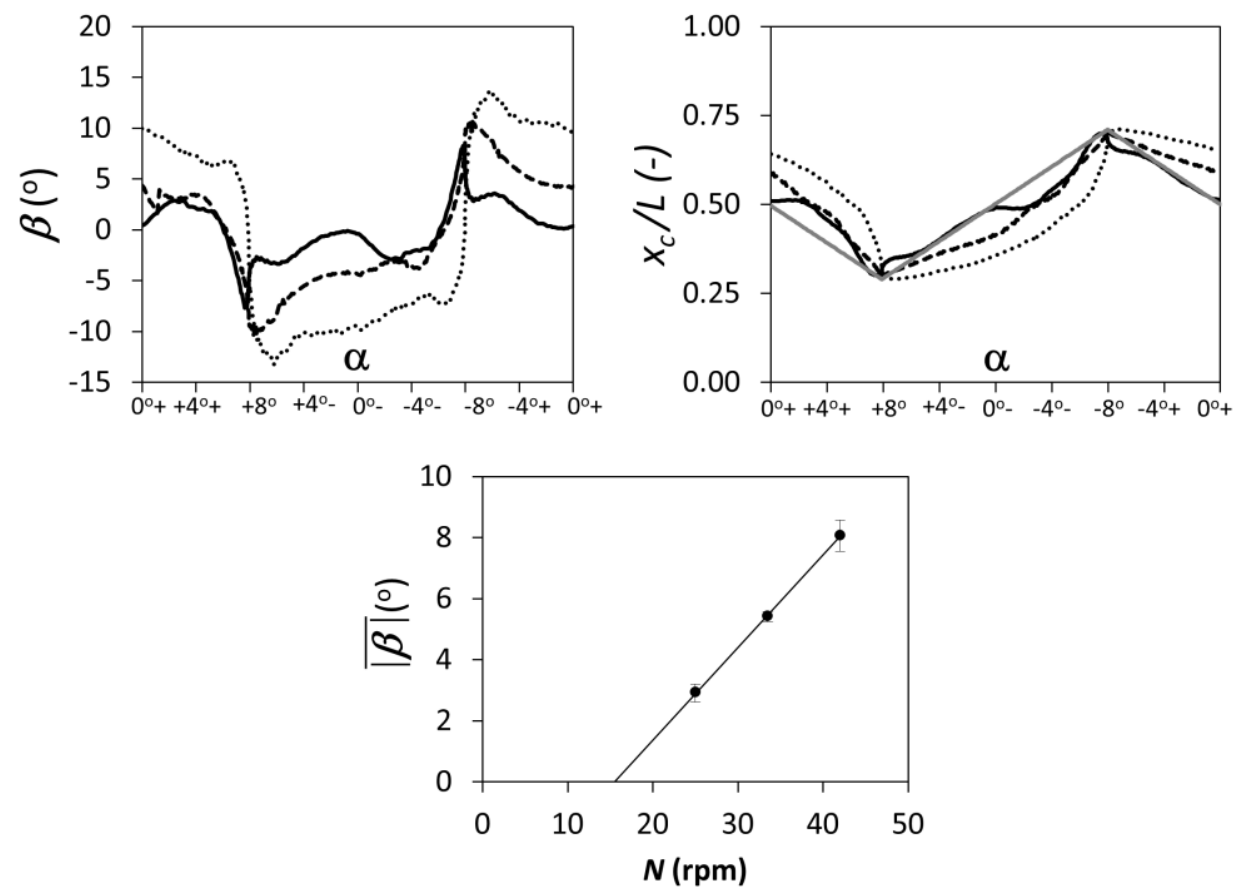

Fig.9 Fluid surface angle (A) and centroid location (B) at different inclinations within one rocking cycle at 25rpm (), 33.5rpm (), 42rpm () and reference velocity estimate ( ). C Magnitude of the average fluid surface angle $(\beta)$ calculated within one rocking cycle. Solid line fitted by linear regression $\left(\mathrm{R}^{2}=0.99\right)$. Error bars represent one standard deviation about the mean $(n=3)$. 

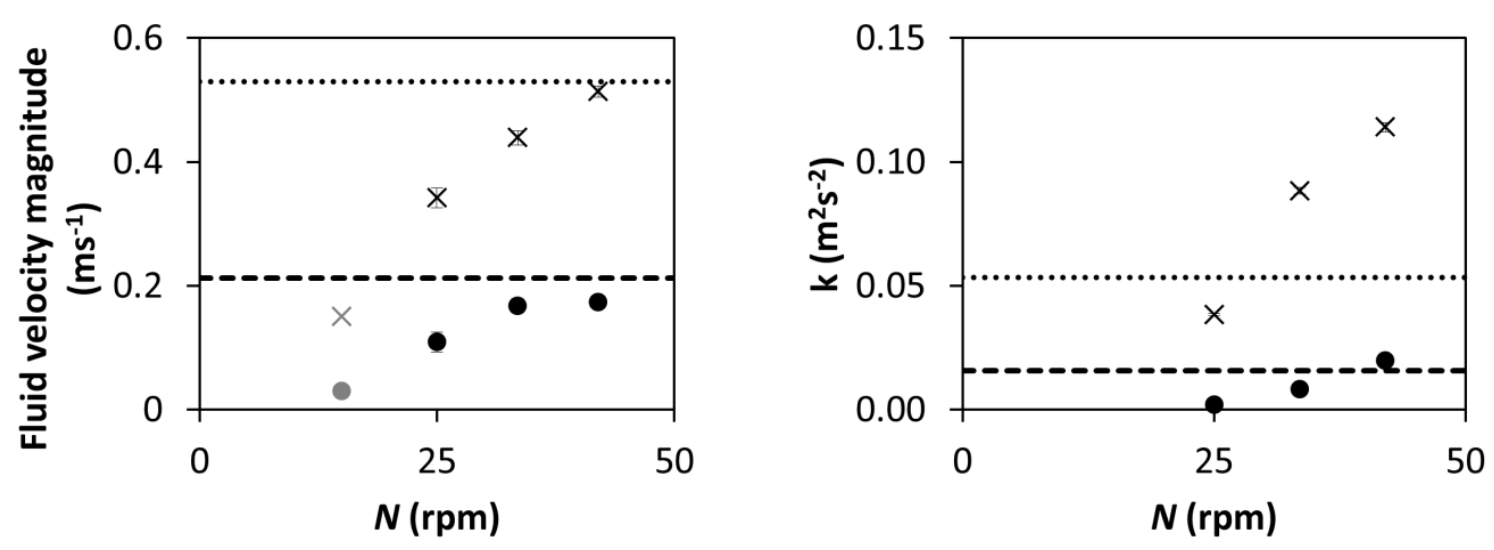

Fig.10 Comparison of average $(\bullet)$ and maximum $(\times)$ velocities and turbulent kinetic energy at different rocking rates. Grey symbols represent data extrapolated from the work of Kalmbach et al., 2011. Dashed and dotted lines represent average and maximum values, respectively, for a 2.7 L stirred tank bioreactor reported by Gabriele et al., 2009. 